\title{
A Rolling Bearing Fault Diagnosis Method Based on EMD and Quantile Permutation Entropy
}

\author{
Qiang-qiang Chen $(\mathbb{D}$, Shao-wu Dai $(\mathbb{D}$, and Hong-de Dai \\ Naval Aviation University, Yantai 264000, China \\ Correspondence should be addressed to Qiang-qiang Chen; 13386386392@163.com
}

Received 7 May 2019; Revised 4 July 2019; Accepted 1 August 2019; Published 4 September 2019

Academic Editor: Frederic Kratz

Copyright (C) 2019 Qiang-qiang Chen et al. This is an open access article distributed under the Creative Commons Attribution License, which permits unrestricted use, distribution, and reproduction in any medium, provided the original work is properly cited.

\begin{abstract}
The vibration signals resulting from rolling bearings are nonlinear and nonstationary, and an approach for the fault diagnosis of rolling bearings using the quantile permutation entropy and EMD (empirical mode decomposition) is proposed. Firstly, the EMD is used to decompose the rolling bearings vibration signal, and several IMFs (intrinsic mode functions) spanning different scales are obtained. Secondly, aiming at the shortcomings of the permutation entropy algorithm, a new permutation entropy algorithm based on sample quantile is proposed, and the quantile permutation entropy of the first few IMFs, which contain the main fault information, is calculated. The quantile permutation entropies are accordingly seen as the characteristic vector and then input to the particle swarm optimization and support vector machine. Finally, the proposed method is applied to the experimental data. The analysis results show that the proposed approach can effectively achieve fault diagnosis of rolling bearings.
\end{abstract}

\section{Introduction}

Rolling bearings are the most frequently used component in a rotary machines, which play an essential role in the modern industry [1]. Rolling bearings failures could lead to uncontrollable losses for industrial equipment [2]. Therefore, rolling bearings fault diagnosis has appealed a significant attention from the research and engineering communities. Rolling bearings fault diagnosis can be summarized in three steps: signal collection, feature extraction, and fault pattern classification [3]. When the rolling bearings work with faults, their fault information always appears to be uncertain, and the vibration signals will also be nonlinear and nonstationary. Therefore, how to extract the operate state from the vibration signals is the key of rolling bearing fault diagnosis [4]. With the application of the classical nonlinear algorithm, a lot of theory, such as chaos, on fractal dimension has been applied to the feature extraction field. Correlation dimension and Naïve Bayes are combined and used to bearing fault diagnosis by Zhang et al. [5]. Chaotic self-synchronization was applied to ball bearing signals by Yau et al. [6]. All of the classical nonlinear theory have made some accomplishments to fault diagnosis.
However, these algorithms often require a lot of time, and it is vulnerable to noise interference in the application process.

The application of entropy theory provides a new idea for rolling bearing fault diagnosis. Pincus proposed the concept of approximate entropy (ApE) to measure the probability of new patterns in signals according to the characteristics of time series [7]. However, ApE depends heavily on the record length, and its estimated value is uniformly lower than the expected one. In order to overcome the shortcomings of ApE, Richman and Moorman put forward a new form of entropy, sample entropy (SampE) [8]. Nonetheless, the definition of SampE must include a template matching; otherwise, it is meaningless. A new kind of entropy named permutation entropy (PE) was put forward by Bandt and Pompe in 2002 [9]. PE is a new method proposed for detecting the randomicity and dynamic changes of time series, which can be used in the field of fault diagnosis. PE and support vector machine (SVM) are combined and used to bearing fault diagnosis by Zhang et al. [10], and the superiority of PE is proved.

However, due to the complexity of mechanical systems, the randomicity and dynamic changes of the vibration signal behave on different scales, making it necessary to 
analyze the vibration signal with $\mathrm{PE}$ in a multiscale way $[11,12]$. The commonly used signal decomposing method is empirical mode decomposition (EMD), and EMD is a kind of data-driven and adaptive nonlinear signal decomposition method and hence suitable for processing nonlinear signals of rolling bearing [13]. In the calculation of $\mathrm{PE}$, the selection of embedding dimension affects the performance of PE to a certain extend. In order to adaptively complete the embedding dimension selection problem and improve the feature extraction ability of $\mathrm{PE}$, a new kind of entropy named sample quantile PE (SQPE) is defined by combining sample quantile and $\mathrm{PE}$ in this paper. Firstly, the EMD method is used to decompose the vibration signal, and several IMFs spanning different scales are obtained. Secondly, the SQPE of the first few IMF components, which contain the main fault information, is calculated. After extracting feature vectors by EMD-SQPE, the SVM which was optimized by particle swarm optimization (PSO) is used as a classifier. PSO enhances the adaptability and feasibility of SVM and improves the recognition ability of SVM.

Compared with the traditional feature extraction method, this paper solves the problem of embedding dimension selection in the $\mathrm{PE}$ calculation process by SQPE entropy calculation and improves the feature extraction ability of PE at the same scale. The proposed method is applied to the bearing experimental data. The results show that the method can effectively distinguish the fault type of rolling bearings and is an effective fault diagnosis method.

The remainder of this paper is organized as follows: Section 2 proposes a concept of SQPE based on PE. In Section 3, the proposed method based on EMD-SQPEPSOSVM is introduced. In Section 4, several examples are presented to demonstrate the effectiveness of the proposed algorithm, and a conclusion is given in Section 5 .

\section{The Proposed Feature Extraction of EMD- SQPE}

In order to realize the feature extraction of different vibration signals of rolling bearings, the multiscale decomposition method is first used to decompose the signals into different time scales. Then, using the SQPE proposed in this paper, the SQPE value of the IMF component containing the main fault information is calculated to deeply mine the rolling bearing fault information.

2.1. Empirical Mode Decomposition. EMD was put forward by Huang N. E. in 1998, which seemed much more promising and had appealed a lot of attention [14]. The EMD algorithm can decompose a nonlinear signal self-adaptively into some IMFs and a residual series [15]:

$$
s_{t}=\sum_{i=1}^{n} \operatorname{imf}_{i}(t)+r_{n}(t)
$$

where $s_{t}$ is the original time series, $\operatorname{imf}_{i}(t)$ is the $i$ th IMF, and $r_{n}(t)$ is the residual series that represents the trend of the signal. The IMF component is a signal that has zero mean and a number of maxima and minima that are equal or differs by at most one.

The EMD algorithm can decompose a nonlinear and nonstationary signal self-adaptively into some IMFs. Each IMF contains fault information of different frequency bands and different scales of the original vibration signal [16].

2.2. Sample Quantile Permutation Entropy (SQPE). The SQPE proposed in this paper is an improved algorithm based on PE, which mainly solves the problem of PE in embedding dimension selection and improves the feature extraction ability of $\mathrm{PE}$ for complex signals through the quantile principle. The definition of SQPE is based on PE, and PE is described as follows.

Consider a time series, $\{x(i), i=1,2, \ldots, N\}$, with length $N$, which can be reconstructed as [17]

$$
\left\{\begin{array}{l}
X_{1}=\{x(1), x(1+\tau), \ldots, x[1+(m-1) \tau]\}, \\
X_{j}=\{x(j), x(j+\tau), \ldots, x[j+(m-1) \tau]\}, \\
\vdots \\
X_{k}=\{x(k), x(k+\tau), \ldots, x[k+(m-1) \tau]\},
\end{array}\right.
$$

where $m$ is the embedding dimension and $\tau$ is the time delay. The element of $X_{j}$ can be rearranged in an increasing order as follows:

$$
x\left[i+\left(j_{1}-1\right) \tau\right] \leq \cdots \leq x\left[i+\left(j_{m}-1\right) \tau\right],
$$

where if there exist two elements in $X_{j}$ that have the same value, such as, $x\left[i+\left(j_{i 1}-1\right) \tau\right]=x\left[i+\left(j_{i 2}-1\right) \tau\right]$, then we order the quantities $x$ according to the values of their corresponding $j ; \quad$ namely, if $j_{i 1}<j_{i 2}$, then $x\left[i+\left(j_{i 1}-1\right) \tau\right] \leq x\left[i+\left(j_{i 2}-1\right) \tau\right]$ is written. Accordingly, any vector $X_{j}$ can be mapped onto a group of symbols as

$$
S_{l}=\left\{j_{1}, j_{2}, \ldots, j_{m}\right\},
$$

where $l=1,2, \ldots, k, k \leq m ! n !$ is the largest number of distinct symbols, and $S_{l}$ is one of the $m$ ! permutations of $m$ distinct symbols, which is mapped onto the $m$ number symbols in $m$-dimensional embedding space. Then, the PE for the time series can be defined as the Shannon entropy for

$$
H_{p}(m)=-\sum_{j=1}^{k} P_{j} \ln \left(P_{j}\right) .
$$

It is noticed that $H_{p}(m)$ attains the maximum value, $\ln (m !)$, when $P_{j}=1 / m !$. For convenience, $H_{p}(m)$ can be normalized by $\ln (m !)$ as

$$
H_{p}=\frac{H_{p}(m)}{\ln (m !)} .
$$

Obviously, the values of $H_{p}$ is $[0,1]$. A bigger value of $H_{p}$ indicates that the signal is much more complex and the maximum value of $H_{p}=1$ means that the signal is very 
nonlinear and nonstationary. Therefore, $\mathrm{PE}$ is a very suitable tool for describing the local order structure and amplifying the dynamic changes of signal.

There are two parameters to be set in the calculation of PE: the embedding dimension $m$ and time delay $\tau$. The time delay $\tau$ has a few effect on the calculation of PE; that is, there are very small differences among the PEs between different time delay $\tau$. So we set $\tau=1$. The embedding dimension $m$ has a big effect on the estimation of PE, and there are differences among the PEs between different embedding dimension $m$. There is no definite standard for the choice of embedding dimension $m$. Bandt recommended $m=2 \sim 7$, and this recommendation is not adaptive because different $m$ values will effect the fault diagnosis results to some extent.

In order to improve the adaptability of the selection with embedding dimension and improve the feature extraction ability of PE. On the basis of PE, this paper introduces the concept of sample quantile and defines SQPE. Sample quantile divides the probability distribution range of a random variable into equal numerical points, without considering the time dimension characteristics of the time series, and directly and effectively characterizes the characteristics of the fluctuation of the signal time series data [18].

Given a time series $X_{1}, X_{2}, \ldots, X_{n}$, define the order statistic as $X_{(1)} \leq X_{(2)} \leq \ldots X_{(n)}$. The $p$ quantile of the time series can be defined as follows [19]:

$$
\left\{\begin{array}{l}
m_{p}=X_{(k)}, \\
k=[n p]+1,
\end{array}\right.
$$

where [.] represents integer operational symbols. $p$ is often set to $0.25,0.5$, and 0.75 . In this paper, we set $p=0.75$.

The sample quantile has the advantages of simple calculation and small calculation. And the sample quantile is a nonparametric statistic, which can accurately reflect the aggregation characteristics of the data at a certain quantile in the absence of the overall distribution prior information. The SQPE is defined as follows:

(1) Given a time series $x(i)$, the PE is calculated under embedding dimension $m=2,3, \ldots, 7$ and $\tau=1$, and their $\mathrm{PE}$ values are denoted by $\mathrm{PE}=\left[\mathrm{PE}_{m=2}\right.$, $\left.\mathrm{PE}_{m=3}, \ldots, \mathrm{PE}_{m=7}\right]$

(2) The 0.75 sample quantile of $\mathrm{PE}=\left[\mathrm{PE}_{m=2}\right.$, $\left.\mathrm{PE}_{m=3}, \ldots, \mathrm{PE}_{m=7}\right]$ is calculated, which is denoted by $\mathrm{SQPE}=$ quantile $(\mathrm{PE})_{p=0.75}$

(3) SQPE is used instead of PE as the feature information for fault diagnosis

The proposed SQPE algorithm can be described briefly as in Figure 1.

In the calculation of SQPE, the problem of embedding dimension selection is not considered, but the entropy value is solved by calculating the sample quantile of all embedding dimensions. This avoids the effect of embedding dimension on feature extraction to some extent.

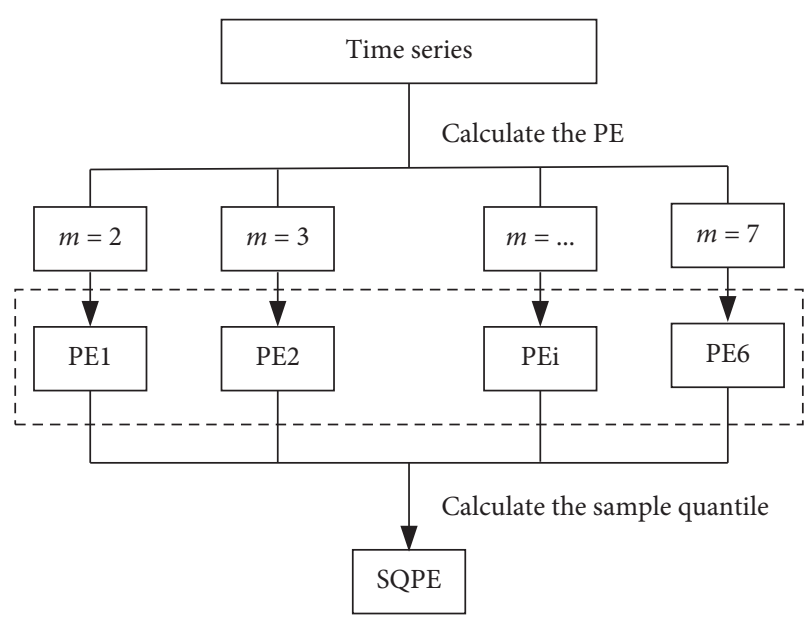

FIgURE 1: Flow chart of SQPE.

\section{The Proposed Fault Diagnosis Based on SQPE}

In addition to EMD-SQPE, the proposed fault diagnosis also adopts the PSO-SVM algorithm. The SVM was originally a deterministic algorithm for finding the linear separating hyperplane of a binary labeled dataset. Similar with the neural network, it has machine learning features. The core algorithm of SVM is to change the original data space to a high-dimensional feature space through a nonlinear mapping [20]. The ability of SVM classification depends largely on the kernel function parameter and penalty parameter. The PSO has the following advantages over other techniques. PSO has the characteristics of stable convergence, and it can get a high-quality solution within shorter calculation time. PSO was applied into adaptive selection of the best penalty parameter and kernel function parameter with $K$-fold cross validation error minimum as the optimization goal [21].

Since the introduction of the PSO algorithm in 1995, researchers have put much effort to improve the original version of PSO. Shi and Eberhart proposed the idea of inertia weight in order to balance the local and global search during the optimization process, in which the inertia weight is linearly decreasing over iterations [22]. The performance of SVM can be improved by optimizing the parameters of SVM through PSO.

Based on the advantages of EMD, SQPE, and PSO-SVM, the proposed rolling bearing fault diagnosis algorithm is described as follows.

(1) Decomposing the initial rolling bearing vibration signals into several IMF components using the EMD method.

(2) SQPE of the first several IMF components which contain the main failure information is calculated and taken as the feature vector. According to [23], the main fault information is often contained in the first few IMFs. The cross correlation coefficients of several IMFs with the original signal are relatively large and retain more information of the original signal. Therefore, the IMFs having large cross 


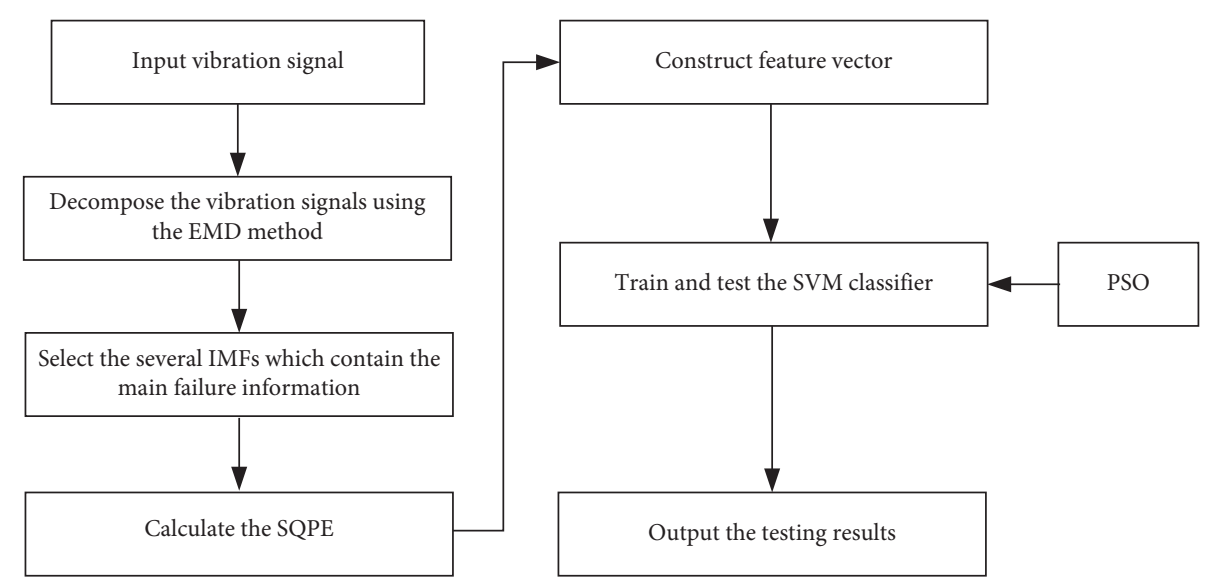

FIgURE 2: Flow chart of the proposed method.

correlation coefficients are used as the main components. The cross correlation can be defined as

$$
\rho_{x y}=\frac{C_{x y}}{\sigma_{x} \sigma_{y}},
$$

where $C_{x y}$ is the cross-covariance function. The cross correlation coefficients $\rho_{x y}$ indicate the correlation between signals; the larger the value of $\rho_{x y}$, the stronger the correlation between two signals.

(3) The feature vectors are seen as the inputs to the SVM classifier. Here, the feature information in (2) is divided into two parts: one is for training, and the other is for testing.

(4) PSO is applied into adaptive selection of the best penalty parameter and kernel function parameter.

(5) The output results of testing samples achieve to discriminate the fault categories automatically.

The proposed EMD-SQPE-PSOSVM fault diagnosis algorithm can be described briefly as in Figure 2.

\section{Simulation Results}

4.1. Experimental Data. In order to validate the capability of the EMD-SQPE-PSOSVM method, experimental analyses on rolling bearing faults were conducted. The 6205-2RS JEM SKF deep groove ball bearing was used in the experimental, and single point faults were introduced to the test rolling bearings using electrodischarge machining with fault diameters of $0.3556 \mathrm{~mm}$ [24]. The shaft rotating speeds of the bearing is $1772 \mathrm{r} / \mathrm{min}$, and the sampling frequency is $12000 \mathrm{~Hz}$. Bearing fault under consideration covers outer race fault (ORF), inner race fault (IRF), rolling element fault (REF), and the norm (NORM), and the length of the bearing vibration signal series is 2048 . There are 80 vibration signals for each category and totally 320 chosen randomly from the data sets. In test 1 3, among these 80 data, 40 samples are randomly selected as training data, and the rest 40 will be seen as testing data.
In the experiments, the feature vectors are seen as the inputs to the PSOSVM model, and the output of the PSOSVM model is the label value corresponding to each fault state, set to NORM (label1), IRF (label2), REF (label3), and ORF (label4).

\subsection{Results}

4.2.1. Test 1: PE(SQPE)-SVM and PE(SQPE)-PSOSVM. $P E$ of the data sets is calculated and taken as the feature vector. The embedding dimension and the delay of PE were set to $m=6$ and $\tau=1.40$ samples of every category are used to train the SVM and PSOSVM classifiers. The classification results of the desired outputs and the outputs of the trained SVM are shown in Figure 3(a). And the results of PSOSVM are shown in Figure 3(b).

The accuracy of 160 testing sets with the feature vector consisting of PE by the SVM classifier is $84.375 \%(135 / 160)$, and the accuracy of the PSOSVM classifier is $87.5 \%$ (140/ $160)$. The best penalty parameter is 1.8232 , and the best kernel function parameter is 645.2294 after PSO optimized.

In order to visually explain the fault diagnosis results of $\mathrm{PE}$ and SQPE, SQPE of the data sets is calculated and taken as the feature vector. The accuracy of 160 testing sets with the feature vector consisting of SQPE by the SVM classifier is $98.125 \%$ (157/160), and the accuracy of the PSOSVM classifier is $98.75 \%$ $(158 / 160)$. The best penalty parameter is 0.1 , and the best kernel function parameter is 2796.8326 after PSO optimized.

4.2.2. Test 2: EMD-PE-SVM and EMD-PE-PSOSVM. As shown in Figure 3, the fault categories cannot be classified from each other. So it is necessary to preprocess the original vibration signals by using EMD. The original signal is decomposed into several IMFs by using the EMD method, respectively. Since the main fault feature is often contained in the first few IMFs, the PE of first five IMFs are calculated and seen as the feature vector and used to train and test the SVM and PSOSVM models. The embedding dimension and the delay of PE were set to $m=6$ and $\tau=1$. The classification results of the desired outputs and the outputs of the trained SVM are shown in Figure 4(a). And the results of PSOSVM are shown in Figure 4(b). 


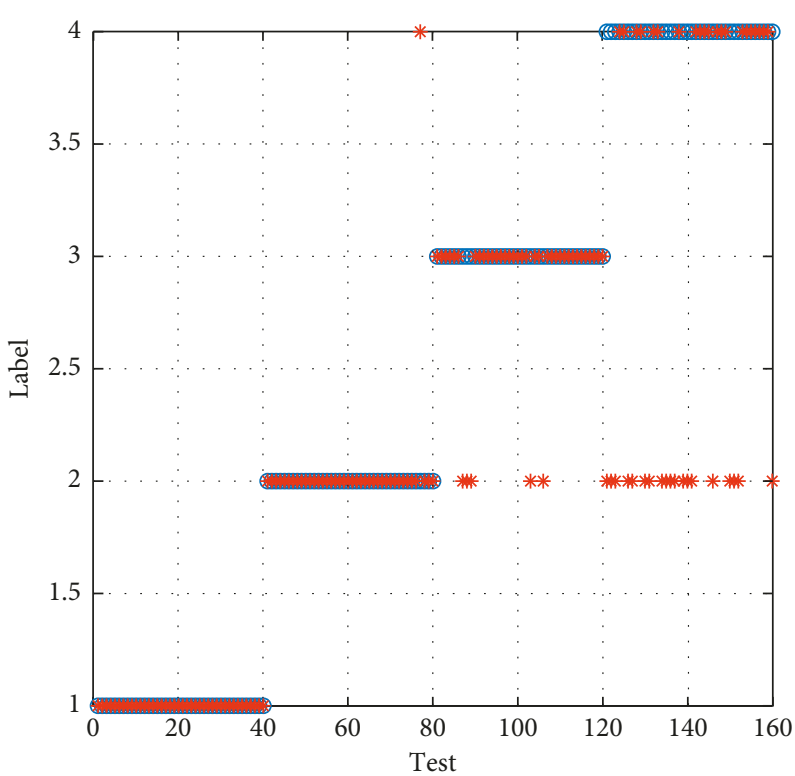

○ True

* Predict

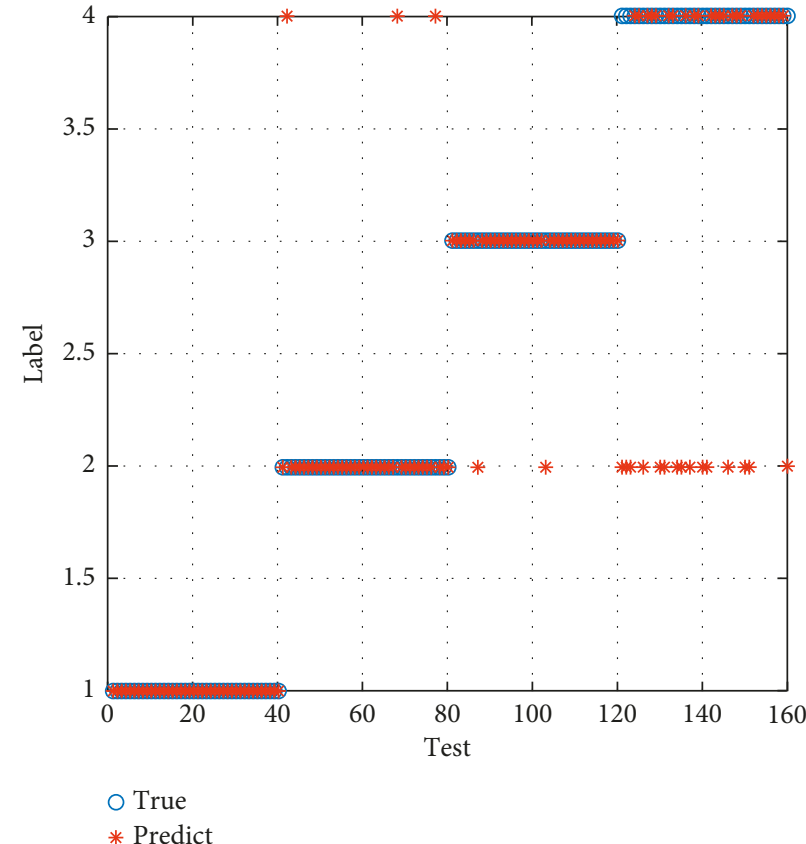

(b)

FIgURE 3: The output results and the desired outputs of testing sets with feature vector consisting of the original data's PE: (a) SVM diagnosis results; (b) PSOSVM diagnosis results.

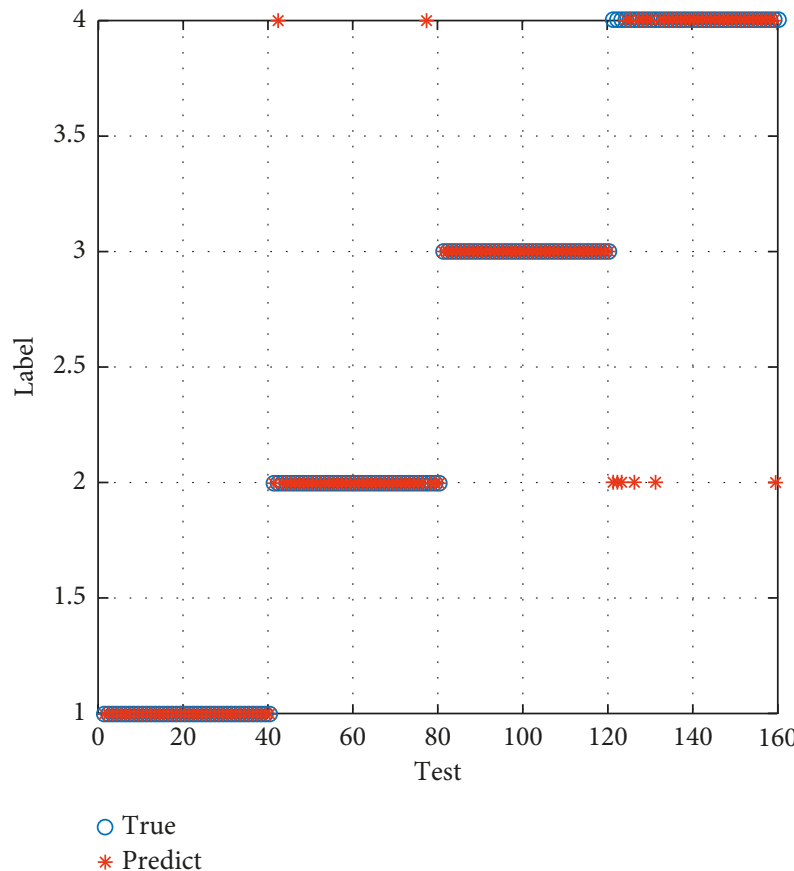

(a)

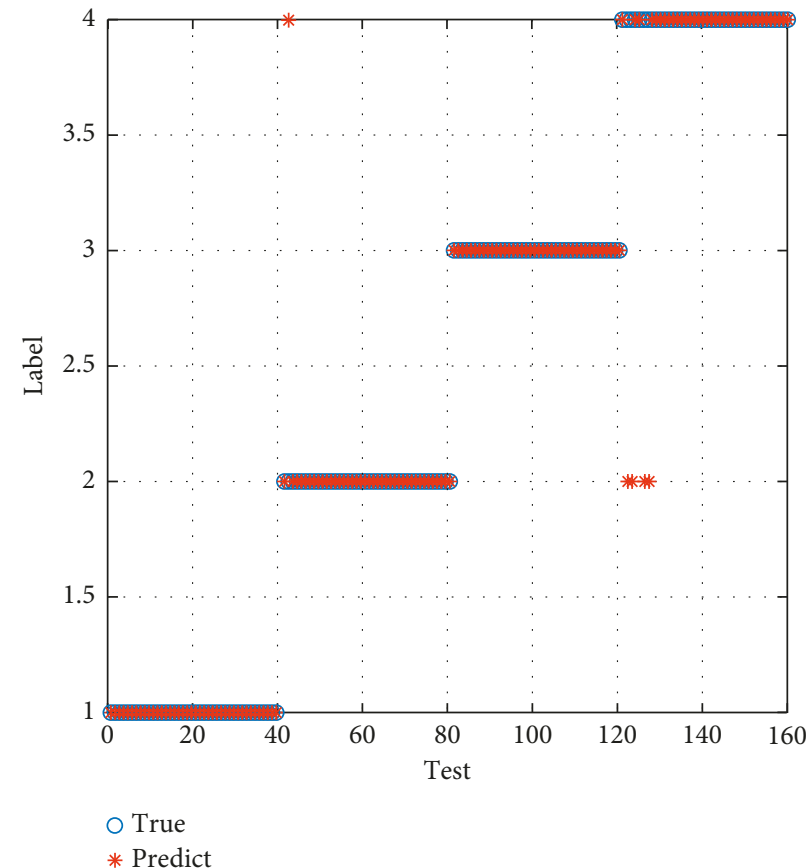

(b)

FIGURE 4: The output results and the desired outputs of testing sets with feature vector consisting of the EMD-PE: (a) SVM diagnosis results; (b) PSOSVM diagnosis results.

The accuracy of 160 testing sets with the feature vector consisting of EMD-PE by the SVM classifier is 95\% (152/160), and the accuracy of the PSOSVM classifier is $96.875 \%$ (155/ 160 ). The best penalty parameter is 13.2931 , and the best kernel function parameter is 1960.7666 after PSO optimized.
4.2.3. Test 3: EMD-SQPE-SVM and EMD-SQPE-PSOSVM. As shown in Figure 4, the accuracy of EMD-PE is obviously higher than PE, but it still cannot be fully classified. Using the EMD-SQPE feature extraction method presented in this paper, the original signal is decomposed into several IMFs by 


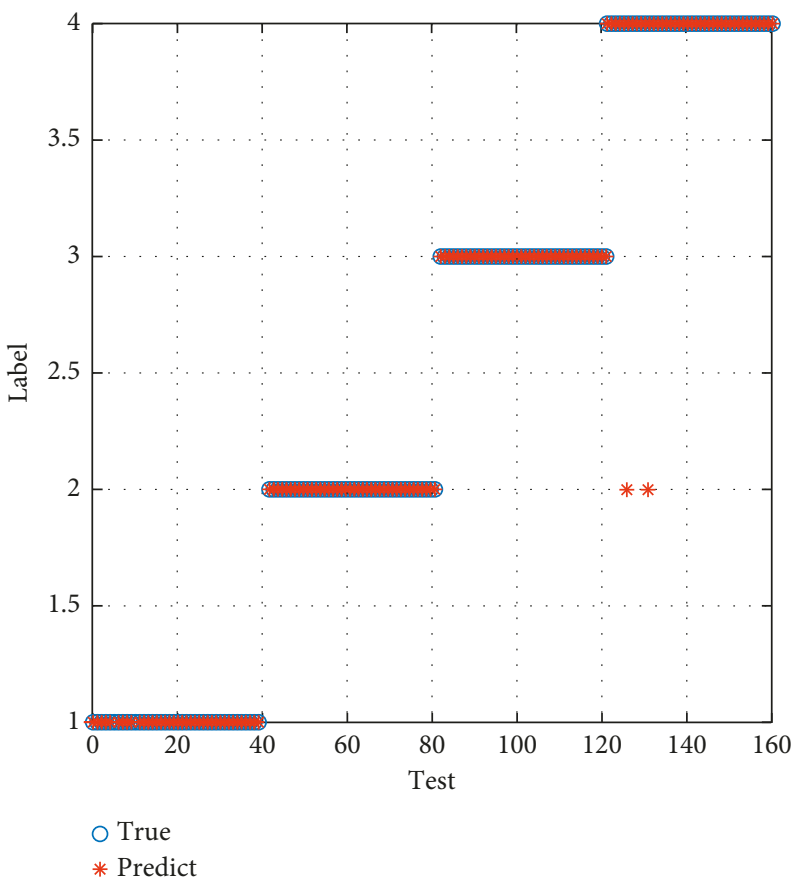

(a)

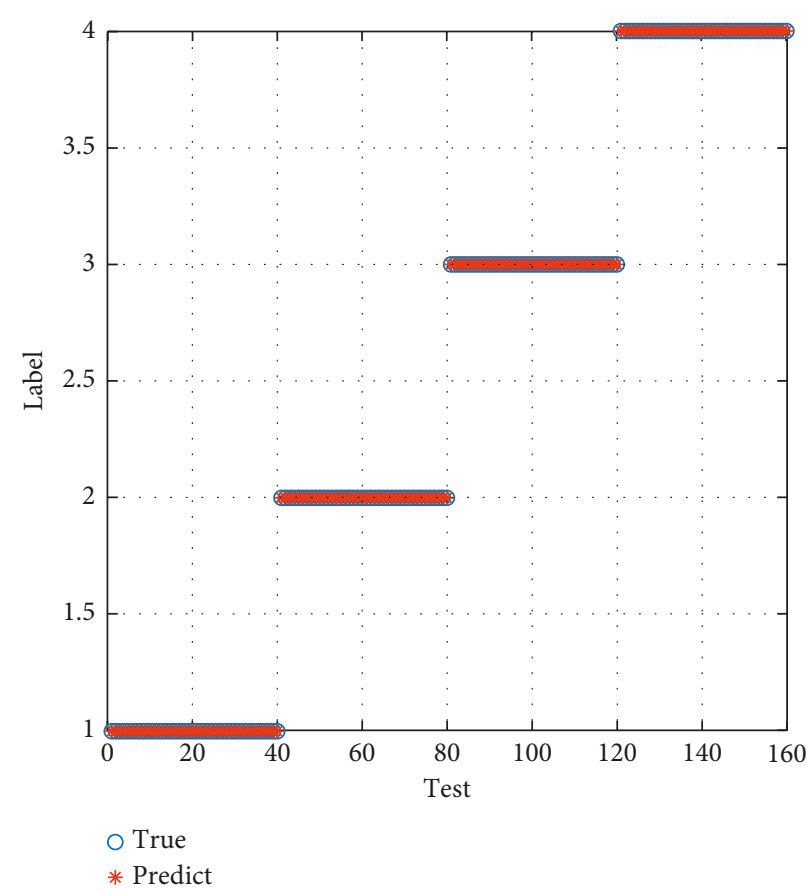

(b)

FIGURE 5: The output results and the desired outputs of testing sets with the feature vector consisting of the EMD-SQPE: (a) SVM diagnosis results; (b) PSOSVM diagnosis results.

TABle 1: Accuracy rate comparison of three algorithms.

\begin{tabular}{|c|c|c|c|c|c|}
\hline Fault diagnosis method & NORM & IRF & REF & ORF & Accuracy (\%) \\
\hline PE-SVM & 40 & 39 & 35 & 21 & 84.375 \\
\hline PE-PSOSVM & 40 & 37 & 38 & 25 & 87.5 \\
\hline EMD-PE-SVM & 40 & 38 & 40 & 34 & 95 \\
\hline EMD-PE-PSOSVM & 40 & 39 & 40 & 36 & 96.875 \\
\hline EMD-SQPE-SVM & 40 & 40 & 40 & 38 & 98.75 \\
\hline EMD-SQPE-PSOSVM & 40 & 40 & 40 & 40 & 100 \\
\hline
\end{tabular}

using the EMD method, and the SQPE of first five IMFs is calculated and seen as the feature vector and used to train and test the SVM and PSOSVM model. The delay of SQPE was set to $\tau=1$, and the embedding dimension was set to $m=2,3 \ldots, 7$. The classification results of the desired outputs and the outputs of the trained SVM are shown in Figure 5(a). And the results of PSOSVM are shown in Figure 5(b).

The accuracy of 160 testing sets with the feature vector consisting of EMD-SQPE by the SVM classifier is $98.75 \%$ $(158 / 160)$, and the accuracy of the PSOSVM classifier is $100 \%(160 / 160)$. The best penalty parameter is 0.1 , and the best kernel function parameter is 1307.5593 after PSO optimized.

In order to visually explain the fault diagnosis results on the vibration signal of the rolling bearing, the results obtained by each method are shown in Table 1 . The numerical values in the table indicate the correct classification results obtained by the classification algorithm for the 40 test set samples in each category.

As shown in Table 1, optimized SVM by PSO can use adaptive selection of the best penalty parameter and kernel function parameter, which improved the accuracy rate of fault diagnosis. The fault diagnosis method based on EMD$\mathrm{PE}$ can analyze the vibration signal with $\mathrm{PE}$ in a multiscale way, which improved the accuracy than PE. The fault diagnosis algorithm proposed in this paper has the best effect, and the recognition rate reaches $100 \%$. It proves the superiority of the SQPE algorithm in fault feature extraction and also proves the effectiveness of the algorithm proposed in this paper.

4.2.4. Test 4: Different Percentages of the Samples Used for Training. To illustrate the influence of the number of training sets, the experiments were designed by different training set scales $(10 \%, 20 \%, 30 \%$, and $40 \%$ of total data sets), and the remaining sets are used for test; the accuracies of the EMD-SQPE-PSOSVM and EMD-PE-PSOSVM for different percentages of the samples used for training are presented in Table 2.

As shown in Table 2, the accuracy of EMD-PE-PSOSVM is not good enough to classify different bearing faults than 
TABLE 2: Accuracies with different training set scales.

\begin{tabular}{|c|c|c|c|c|c|}
\hline \multirow{2}{*}{ Fault diagnosis method } & \multicolumn{5}{|c|}{ Percentage of the samples used for training } \\
\hline & $10 \%$ & $20 \%$ & $30 \%$ & $40 \%$ & $50 \%$ \\
\hline EMD-PE-PSOSVM & $97.9167 \%$ & $96.875 \%$ & $97.7679 \%$ & $97.3958 \%$ & $96.875 \%$ \\
\hline EMD-SQPE-PSOSVM & $100 \%$ & $100 \%$ & $100 \%$ & $100 \%$ & $100 \%$ \\
\hline
\end{tabular}

EMD-SQPE-PSOSVM with different percentages of the samples used for training. Even with less training data sets, the EMD-SQPE-PSOSVM still has 100\% accuracy.

\section{Conclusion}

In this paper, a new rolling bearing fault diagnosis method has been introduced based on the vibration signal analysis using EMD-SQPE and PSOSVM. The test data analysis conclusions illustrate that the proposed algorithm can not only analyze among different fault categories but also identify the level of fault severity. The experimental results show the following:

(1) SQPE is a valid method to measure the complexity of nonlinear and nonstationary vibration signals. Compared with PE, SQPE can extract the features with high distinguishability.

(2) SQPE can avoid the selection of embedding dimension, and it is not necessary to determine the specific embedding dimension in the solution process, which has better adaptability.

(3) EMD has a good application prospect in analyzing the nonlinear signal. After the decomposition of the original signal by EMD, entropy is applied to measure the feature of the IMFs obviously.

(4) PSO can select the best penalty parameter and kernel function parameter adaptively, which improved the performance of SVM.

\section{Data Availability}

The data used to support the findings of this study are available from the corresponding author upon request.

\section{Conflicts of Interest}

The authors declare that they have no conflicts of interest.

\section{Acknowledgments}

This research was funded by the Shandong Natural Science Foundation of China (grant number ZR2017MF036) and Defense Science and Technology Project Foundation of China (grant number F062102009).

\section{References}

[1] H. Wang, Y. Ke, L. Song, G. Tang, and P. Chen, "A sparsitypromoted decomposition for compressed fault diagnosis of roller bearings," Sensors, vol. 16, no. 9, pp. 1524-1532, 2016.
[2] M. Yasir and B.-H. Koh, "Data decomposition techniques with multi-scale permutation entropy calculations for bearing fault diagnosis," Sensors, vol. 18, no. 4, p. 1278, 2018.

[3] S.-D. Wu, P.-H. Wu, C.-W. Wu, J.-J. Ding, and C.-C. Wang, "Bearing fault diagnosis based on multiscale permutation entropy and support vector machine," Entropy, vol. 14, no. 8, pp. 1343-1356, 2012.

[4] F. Wang, Y. Zhang, B. Zhang, and W. Su, "Application of wavelet packet sample entropy in the forecast of rolling element bearing fault trend," in Proceedings of the 2011 International Conference on Multimedia and Signal Processing, Guilin, China, May 2011.

[5] N. Zhang, L. Wu, J. Yang, and Y. Guan, "Naive Bayes bearing fault diagnosis based on enhanced independence of data," Sensors, vol. 18, no. 2, p. 463, 2018.

[6] H. T. Yau, S. Y. Wu, C. L. Chen, and Y. C. Li, "Fractionalorder chaotic self-synchronization-based tracking faults diagnosis of ball bearing systems," IEEE Transactions on Industrial Electronics, vol. 63, no. 6, pp. 3824-3833, 2016.

[7] S. M. Pincus, "Approximate entropy as a measure of system complexity," Proceedings of the National Academy of Sciences, vol. 88, no. 6, pp. 2297-2301, 1991.

[8] J. S. Richman and J. R. Moorman, "Physiological time-series analysis using approximate entropy and sample entropy," American Journal of Physiology-Heart \& Circulatory Physiology, vol. 278, no. 6, pp. H2039-H2049, 2000.

[9] C. Bandt and B. Pompe, "Permutation entropy: a natural complexity measure for time series," Physical Review Letters, vol. 88, no. 17, article 174102, 2002.

[10] X. Zhang, Y. Liang, J. Zhou, and Y. Zang, "A novel bearing fault diagnosis model integrated permutation entropy, ensemble empirical mode decomposition and optimized SVM," Measurement, vol. 69, pp. 164-179, 2015.

[11] J. Zheng, J. Cheng, and Y. Yang, "Multiscale permutation entropy based rolling bearing fault diagnosis," Shock and Vibration, vol. 2014, Article ID 154291, 8 pages, 2014.

[12] K. Li, L. Su, J. Wu, H. Wang, and P. Chen, "A rolling bearing fault diagnosis method based on variational mode decomposition and an improved kernel extreme learning machine," Applied Sciences, vol. 7, no. 10, p. 1004, 2017.

[13] N. E. Huang, Z. Shen, S. R. Long et al., "The empirical mode decomposition and the Hilbert spectrum for nonlinear and non-stationary time series analysis," Proceedings of the Royal Society of London. Series A: Mathematical, Physical and Engineering Sciences, vol. 454, no. 1971, pp. 903-995, 1998.

[14] M. Li, H. Wang, G. Tang, H. Yuan, and Y. Yang, "An improved method based on CEEMD for fault diagnosis of rolling bearing," Advances in Mechanical Engineering, vol. 6, article 676205, 2014

[15] N. I. A. Rashid, A. Shabri, and R. Samsudin, "New hybridization of empirical mode decomposition and least squares support vector machine model in forecasting Malaysia exchange rates," in Proceedings of the 2017 International Conference on Robotics, Automation and Sciences (ICORAS), pp. 1-5, IEEE, Melaka, Malaysia, November 2017. 
[16] D. M. Klionskiy, D. I. Kaplun, and V. V. Geppener, "Empirical mode decomposition for signal preprocessing and classification of intrinsic mode functions," Pattern Recognition and Image Analysis, vol. 28, no. 1, pp. 122-132, 2018.

[17] C. Bandt, "Permutation entropy and order patterns in long time series," in Time Series Analysis and Forecasting, pp. 61-73, Springer, Berlin, Germany, 2016.

[18] M. I. Salazar-Alvarez, C. Temblador-Pérez, W. J. Conover, V. G. Tercero-Gómez, A. E. Cordero-Franco, and M. G. Beruvides, "Regressing sample quantiles to perform nonparametric capability analysis," The International Journal of Advanced Manufacturing Technology, vol. 86, no. 5-8, pp. 1347-1356, 2016.

[19] C. Jentsch and A. Leucht, "Bootstrapping sample quantiles of discrete data," Annals of the Institute of Statistical Mathematics, vol. 68, no. 3, pp. 491-539, 2016.

[20] M. D. C. Moura, E. Zio, I. D. Lins, and E. Droguett, "Failure and reliability prediction by support vector machines regression of time series data," Reliability Engineering \& System Safety, vol. 96, no. 11, pp. 1527-1534, 2017.

[21] C.-M. Lee and C.-N. Ko, "Time series prediction using RBF neural networks with a nonlinear time-varying evolution PSO algorithm," Neurocomputing, vol. 73, no. 1-3, pp. 449-460, 2009.

[22] E. García-Gonzalo and J. L. Fernández-Martínez, "A brief historical review of particle swarm optimization (PSO)," Journal of Bioinformatics and Intelligent Control, vol. 1, no. 1, pp. 3-16, 2012.

[23] H. Wang, R. Li, G. Tang, H. Yuan, Q. Zhao, and X. Cao, "A compound fault diagnosis for rolling bearings method based on blind source separation and ensemble empirical mode decomposition," PLoS One, vol. 9, no. 10, Article ID e109166, 2014.

[24] W. A. Smith and R. B. Randall, "Rolling element bearing diagnostics using the Case Western Reserve University data: a benchmark study," Mechanical Systems and Signal Processing, vol. 64-65, pp. 100-131, 2015. 


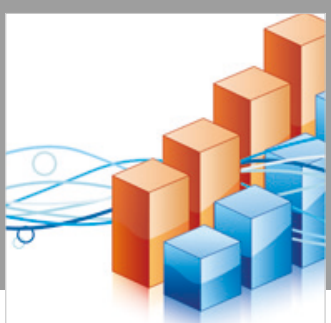

Advances in

Operations Research

\section{-n-m}
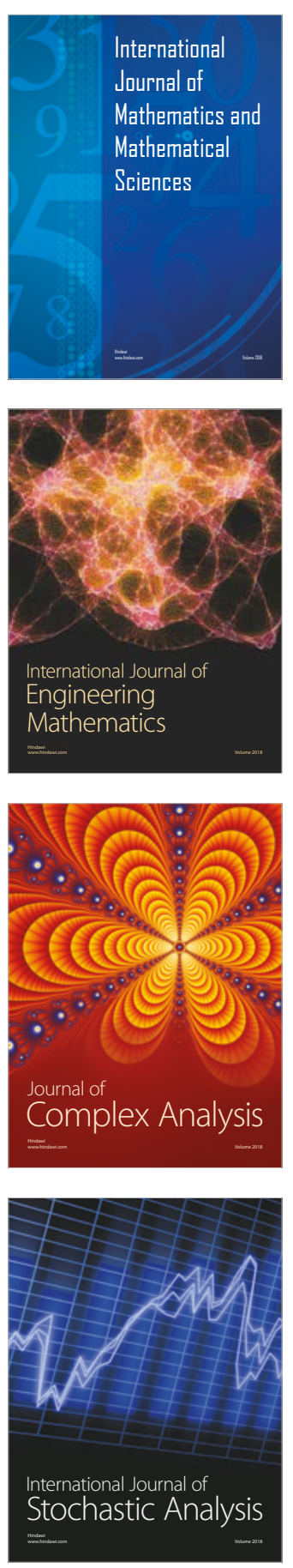
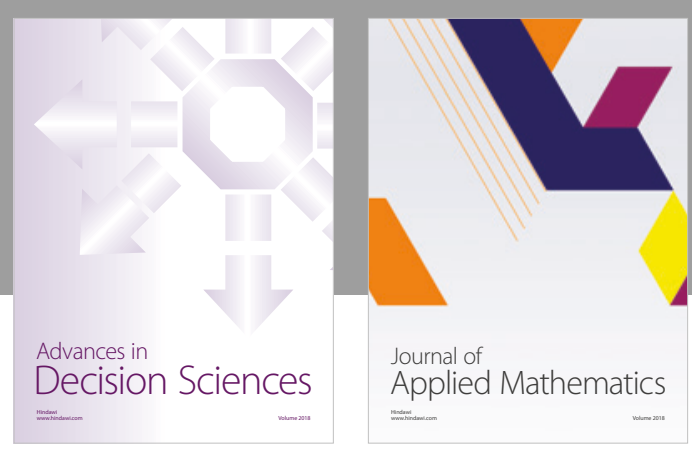

Journal of

Applied Mathematics
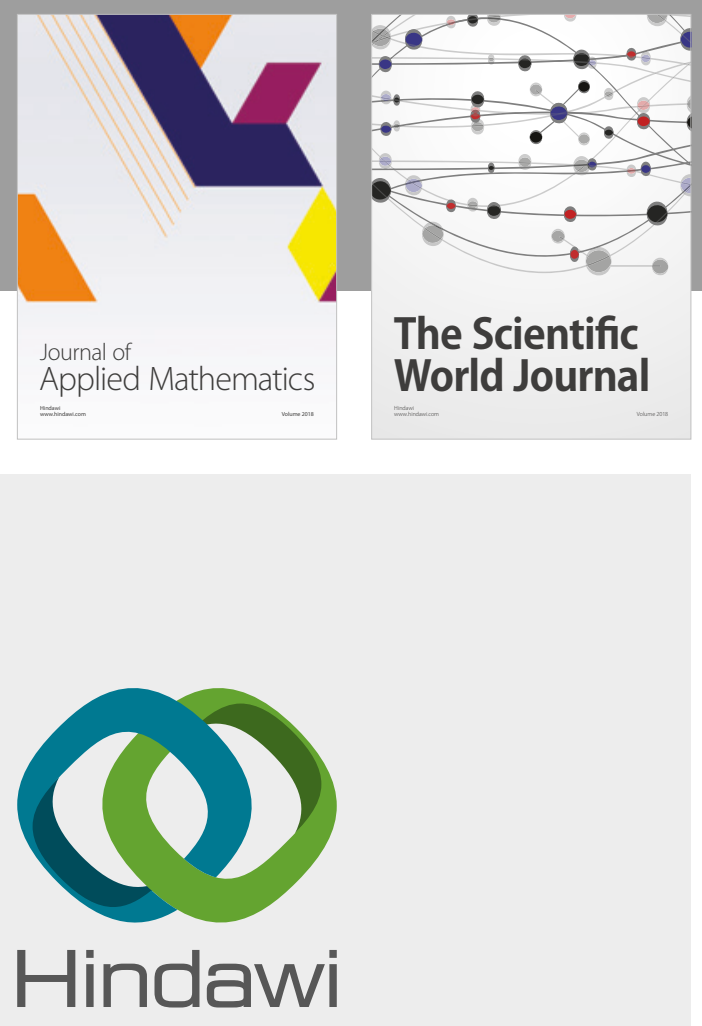

Submit your manuscripts at

www.hindawi.com

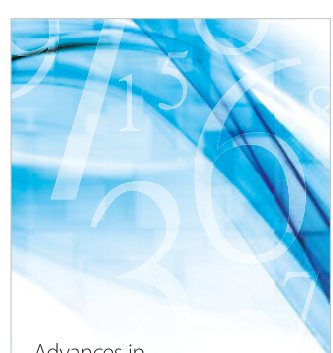

Advances in
Numerical Analysis
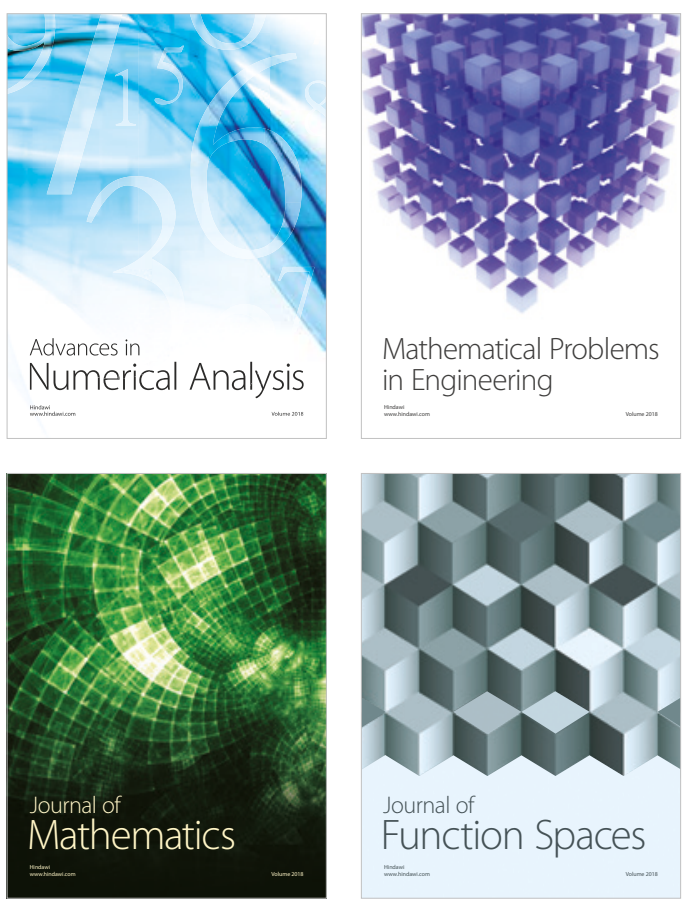

Mathematical Problems in Engineering

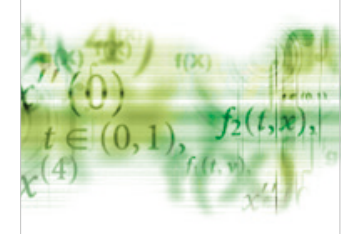

International Journal of

Differential Equations

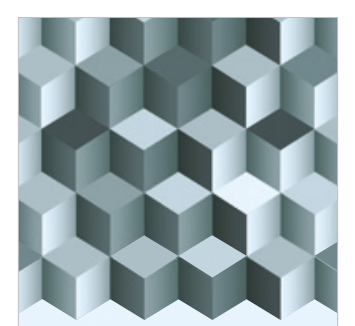

Journal of

Function Spaces
The Scientific

World Journal

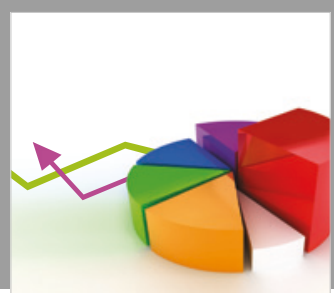

Journal of

Probability and Statistics
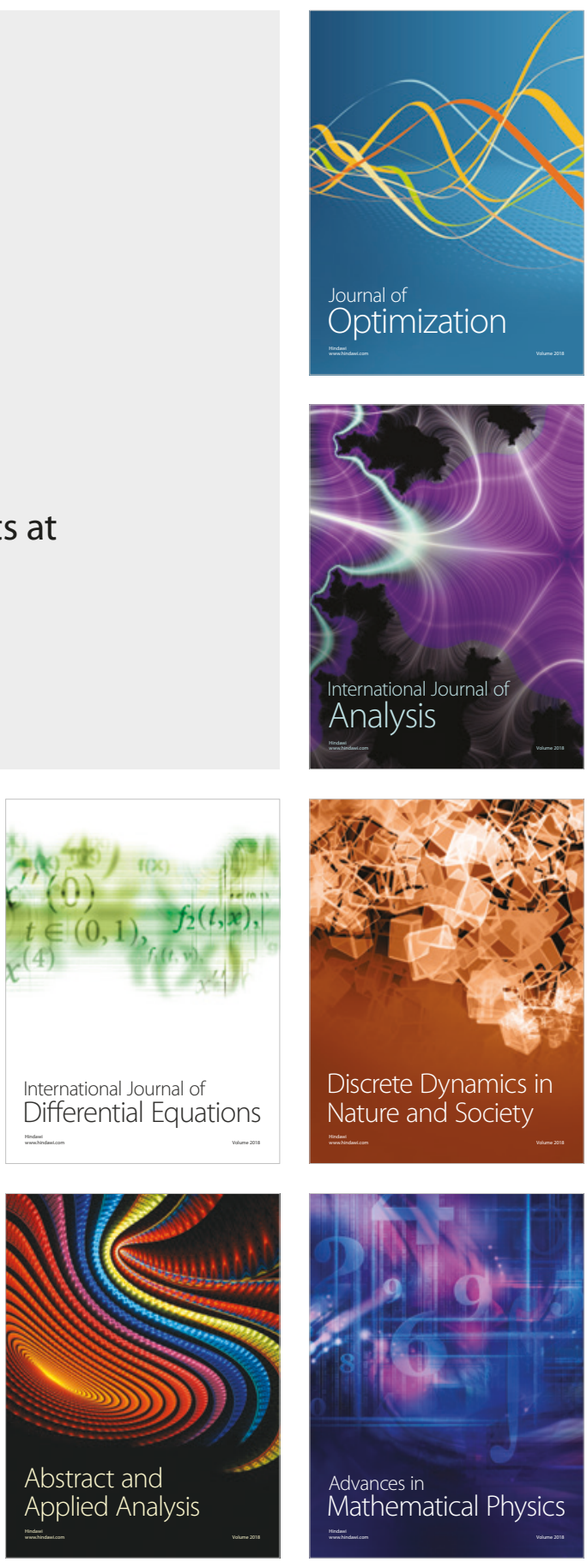